\title{
Thyristor Voltage Regulator Experimental Research
}

\author{
Elena Sosnina ${ }^{1, *}$, Aleksandr Sevostyanov ${ }^{1}$, Evgeny Kryukov $^{1}$, and Rustam Bedretdinov ${ }^{1}$ \\ ${ }^{1}$ Nizhny Novgorod State Technical University n.a. R.E. Alekseev, Department of Electric Power Engineering, Power Supply and Power \\ Electronics, Nizhny Novgorod, Russia
}

\begin{abstract}
The article is devoted to the thyristor voltage regulator (TVR) development. The TVR purpose is to control power flows and regulate voltage in $6-20 \mathrm{kV}$ distribution electrical networks (DEN). The principle of TVR operation is based on the plus EMF (or minus EMF) introduction into power line when the shared use of longitudinal (change of magnitude) and transverse (change of phase) voltage regulation. The description of the TVR prototype is given. The TVR prototype consists of a $0.4 \mathrm{kV}$ thyristor switches, power transformers (shunt and serial) and a $6 \mathrm{kV}$ switchgear. The TVR has a two-level control system (CS). The TVR prototype experimental research was conducted in four stages: check of power equipment, first level CS research, second level CS research, prototype tests as a whole. The connection diagrams (thyristor switches unit, transformer and measuring equipment) and contact connections reliability were checked when the power part was tested. A qualitative characteristic of the input and output signals was obtained when testing the first level CS. It is found that the thyristor control pulses are formed according to the developed algorithm. The correctness of control system algorithms, executed and transmitted commands, passed and received data was confirmed as a result of the second level CS tests. The TVR research results indicate that the prototype provides the smoothness and specified accuracy of voltage regulation in all modes. The control range of the output voltage relative to the input was $\pm 10 \%$. The discreteness of regulation did not exceed $1.5 \%$. The range of change in the shift angle of the output voltage relative to the input was $\pm 5^{\circ}$. Research confirmed the TVR ES operability and its readiness for trial operation.
\end{abstract}

\section{Introduction}

The electric power industry development and its transition to a new technological level are connected with implementation of Internet of energy concept [1,2]. This technology is aimed at converting electric networks from a passive device for transporting and distributing electricity to an active one that ensures energy security and power supply quality [3, 4]. The interaction of distributed electricity sources, its accumulators and active consumers will be carried out on the basis of "horizontal" connections and multi-party services. This approach will preserve the advantages of both centralized and decentralized power supply systems [5, $6]$.

Two important tasks must be solved when building distribution electrical networks (DEN) that operate on the Internet of energy principle: power flows control and power quality ensuring [7]. These tasks can be solved using devices that implement D-FACTS (Distributed Flexible Alternative Current Transmission Systems) technologies. D-FACTS include such devices as an unified power flow controller (UPFC) [8], an interline power flow controller (IPFC) [9], a distributed static series compensator (DSSC) [10], a thyristor controlled phase angle regulator (TCPAR) [11], a thyristor switched series capacitor (TSSC) [12], etc. Currently, these devices are either in development or at the stage of trial operation.

Low power quality in the MV DEN is often caused by voltage deviations. Load tap changers (LTCs) [1-16] and step voltage regulators $[17,18]$ are used to regulate the voltage levels. Booster transformers (BT) are widely used [19]. The main BT disadvantage is their low response which makes them inefficient in electric networks with a dynamic load.

A thyristor voltage regulator (TVR) prototype which was developed by scientists of Nizhny Novgorod state technical university n. a. R.E. Alekseev allows to provide the power flow controlling and to ensure power quality [20].

The principle of TVR operation is based on the shared use of longitudinal (change of magnitude) and transverse (change of phase) voltage regulation. Adding EMF in the line under longitudinal regulation allows to change the voltage level on the consumer's buses. The

\footnotetext{
* Corresponding author: sosnyna@yandex.ru
} 
change in phase of the output voltage under transverse regulation allows to control active and reactive power flow.

The TVR favorably differs in response, switching resource and smooth regulation compared to electromechanical regulators.

The article is devoted to research of TVR prototype operability, the effectiveness of control system, and the readiness of the prototype for trial operation.

\section{Operation principle of thyristor voltage regulator}

Fig. 10 shows a schematic circuit of the thyristor voltage regulator.

The TVR is based on longitudinal and transverse control thyristor modules, shunt and series transformers. The primary windings of the shunt transformer $\mathrm{T} 1$ are delta-connected. The secondary windings of each three phases T1 are made in the form of three galvanically isolated sections.

The sections with EMF1 $\left(e_{1 A}, e_{1 B}, e_{1 C}\right)$ form a threephase voltage system for power supply the transverse control module (TS1-TS4).

The sections with EMF2 $\left(e_{2 A}, e_{2 B}, e_{2}\right)$ form a threephase voltage system for power supply the longitudinal control module (TS5-TS8).

The transverse and longitudinal control modules of each phase are made according to the reversible AC bridge scheme. The bridges diagonals are series connected and form a power supply circuit for the primary windings of serial transformers (T2).

The secondary windings of $\mathrm{T} 2$ are included in the phase dissection of DEN lines. Their voltages are summed with the TVR input voltage.

Thyristor switches placed in the secondary windings of circuit T1 are under low potential. This significantly reduces the requirements for their isolation from constructional elements.

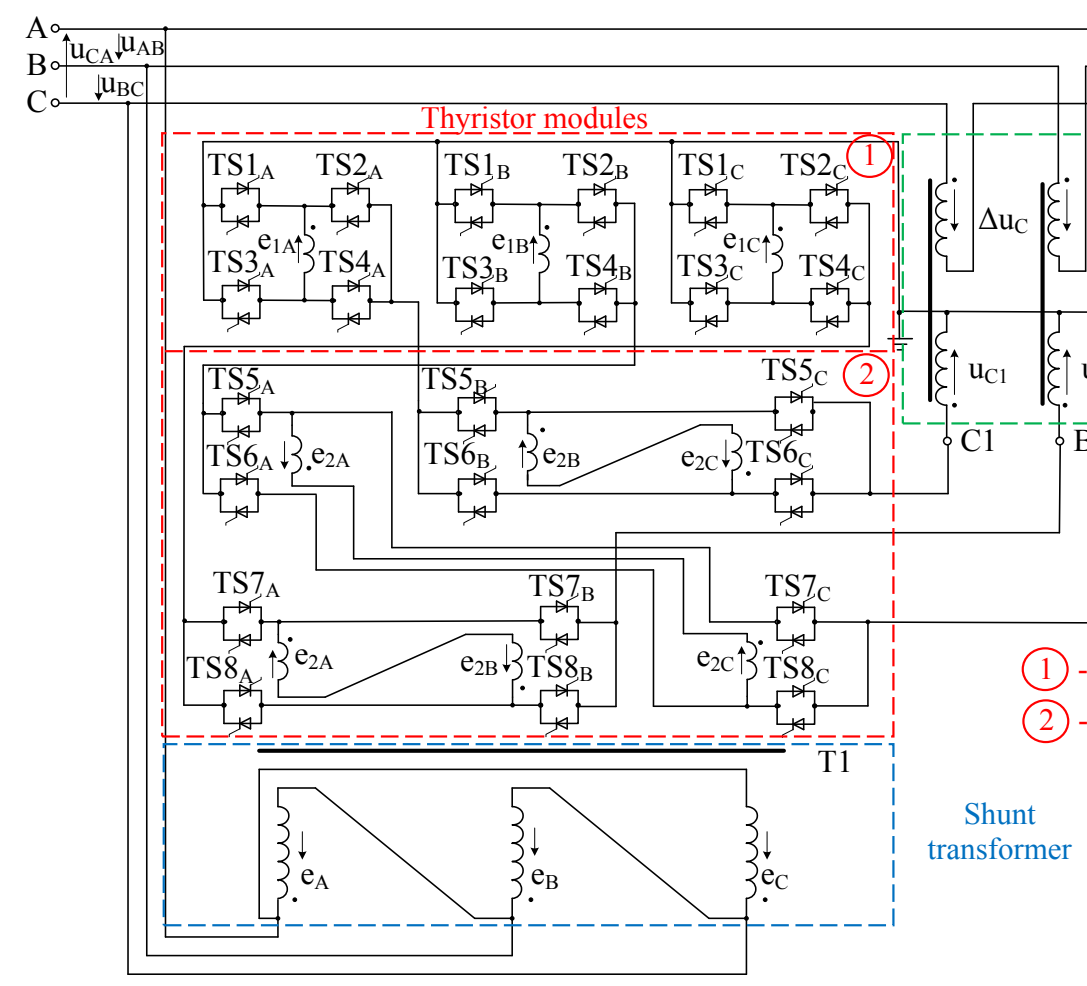

Fig. 1. Schematic diagram of the TVR power part .

It should be noted that using the pulse-phase control principle allows to regulate the value and phase of the TVR output voltage smoothly.

The direct transmission mode of input voltage to the TVR output is implemented when the TS3, TS4 and TS6, TS8 of all phases are switched on. In this case, all secondary windings $\mathrm{T} 2$ are excluded from the supply circuit $\mathrm{T} 1$, and the primary windings $\mathrm{T} 2$ are shorted to neutral, and their voltage, as well as the voltage of the secondary windings $\Delta u$, is zero.

As a result, the TVR output voltages are equal to the corresponding input voltages:

$$
\begin{aligned}
& u_{A 1 B 1}=u_{A 2 B 2} ; \\
& u_{B 1 C 1}=u_{B 2 C 2} ; \\
& u_{C 1 A 1}=u_{C 2 A 2} .
\end{aligned}
$$

The phases of the output voltage are changed by transverse regulation. This allows to control the active and reactive power flows in the power line. In this case voltages are formed shifted by $90^{\circ}$ relative to the phase voltages of the network.

It is possible to implement the delay and advance modes of the TVR output voltage relative to the input. The delay mode is activated when switches TS2, TS3 and TS6, TS8 of all phases are on. At the same time, EMF $e_{1 A}, e_{1 B}, e_{1 C}$, are introduced into the power supply circuit of the primary windings $\mathrm{T} 2$ which differ from the input voltages of the TVR in proportion to the transformation ratio of the transverse control stages $\mathrm{T} 1$ $\left(k_{11}\right)$. It should be noted that the EMF of phase $\mathrm{B}\left(e_{1 B}\right)$ is used for phase A. The EMF of phase $\mathrm{B}\left(e_{1 B}\right)$ is in 
antiphase with the line voltage $u_{B 1 C 1}$. Similarly, the EMF of phases $\mathrm{C}\left(e_{1 C}\right)$ and $\mathrm{A}\left(e_{1 A}\right)$ are used for the $\mathrm{B}$ and $\mathrm{C}$ phases voltages, respectively. These EMFs are in antiphase with the input line voltages $u_{C 1 A 1}, u_{A 1 B 1}$. Accordingly, the addition voltages of the secondary windings $\Delta u_{A п \Pi}, \Delta u_{B \Pi \Pi}, \Delta u_{C п \Pi}$ are introduced into the line. These additional voltages differ from the voltages of the primary windings $u_{A 1}, u_{B 1}, u_{C 1}$ in proportion to the transformation ratio $\left(k_{2}\right) \mathrm{T} 2$ :

$$
\begin{aligned}
& \Delta u_{A \text { пा }}=k_{2} \cdot u_{A 1}=k_{2} \cdot e_{1 B}=-k_{11} \cdot k_{2} \cdot u_{B 1 C 1} ; \\
& \Delta u_{B \text { пा }}=k_{2} \cdot u_{B 1}=k_{2} \cdot e_{1 C}=-k_{11} \cdot k_{2} \cdot u_{C 1 A 1} ; \\
& \Delta u_{C \text { пा }}=k_{2} \cdot u_{C 1}=k_{2} \cdot e_{1 A}=-k_{11} \cdot k_{2} \cdot u_{A 1 B} .
\end{aligned}
$$

The vector diagram of the input and output line voltages for the delay mode is shown in Fig. 2. From the presented diagram it follows that the introduction of a transverse regulation step into the line of each phase allows to obtain linear voltages at the TVR output, lagging in phase relative to the input voltages by an angle $\alpha$.

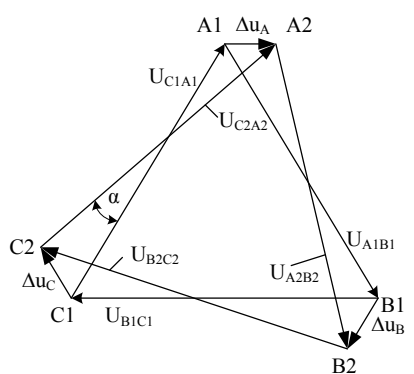

Fig. 2. Voltage vector diagram for the delay mode.

It can be shown that EMF $e_{1 A}, e_{1 B}, e_{1 C}$ are reversed when switches TS1-TS4 and TS6-TS8 of all phases are turned on. Due to this, the mode of advancing the TVR output voltage relative to the input voltage is also realized on the angle $\alpha$.

Change of phase of the output voltage relative to the input one allows to regulate an active and reactive power flows transmitted through the AC power line between two nodes of the DEN which are determined by the formulas:

$$
\begin{gathered}
P=\frac{U_{1} \cdot U_{2}}{x_{P L}} \cdot \sin \delta \\
Q_{1}=\frac{1}{x_{P L}} \cdot\left(U_{1}^{2}-U_{1} \cdot U_{2} \cdot \cos \delta\right) \\
Q_{2}=\frac{1}{x_{P L}} \cdot\left(U_{2}^{2}-U_{1} \cdot U_{2} \cdot \cos \delta\right)
\end{gathered}
$$

where $U_{1}$ and $U_{2}$ - voltages of a power line $(P L)$ initial and in the end; $Q_{1}$ и $Q_{2}$ - reactive power of a PL initial and in the end; $x_{P L}$ - power line reactance; $\delta$ - angle between the $U_{1}$ и $U_{2}$ voltage.

The longitudinal control modules are powered by secondary windings $\mathrm{T} 1$ with EMF values $e_{2 A}, e_{2 B}, e_{2 C}$. Moreover, the EMF of the secondary windings $e_{2 A}, e_{2 B}$, $e_{2 C}$ are in antiphase with the input line voltages of the TVR $u_{A 1 B 1}, u_{B 1 C 1}, u_{C 1 A 1}$ and differ from them in magnitude in proportion to the transformation ratio $\left(k_{12}\right)$.

When the switches $\mathrm{TS}_{\mathrm{A}}$, TS7 $\mathrm{C}$ and TS3, TS4 of all phases are switched on, the EMF difference of the longitudinal control stages $e_{2 C}-e_{2 A}$ is introduced into the power supply chain of phase A of the primary winding T2. Accordingly, when the switches $\mathrm{TS} 8_{A}, \mathrm{TS} 7_{B}$ and $\mathrm{TS} 6_{B}, \mathrm{TS} 5_{C}$ are turned on, the differences in the EMF $e_{2 A}-e_{2 B}$ and $e_{2 B}-e_{2 C}$ are introduced in the supply circuit of phases $B$ and $C$ T2. Thus, the voltage additions of the secondary windings $\Delta u_{A l o n}, \Delta u_{B l o n}, \Delta u_{C l o n}$, which differ from the specified geometric difference in proportion to the T2 transformation ratio $k_{2}$, are introduced into the series of the TVR line:

$$
\begin{gathered}
\Delta u_{\text {Alon }}=k_{2} \cdot u_{A 1}=k_{2} \cdot\left(e_{2 C}-e_{2 A}\right)=k_{12} \cdot k_{2} \cdot\left(u_{A 1 B 1}-u_{C 1 A 1}\right) ; \\
\Delta u_{\text {Blon }}=k_{2} \cdot u_{B 1}=k_{2} \cdot\left(e_{2 A}-e_{2 B}\right)=k_{12} \cdot k_{2} \cdot\left(u_{B 1 C 1}-u_{A 1 B 1}\right) ; \\
\Delta u_{C l o n}=k_{2} \cdot u_{C 1}=k_{2} \cdot\left(e_{2 B}-e_{2 C}\right)=k_{12} \cdot k_{2} \cdot\left(u_{C 1 A 1}-u_{B 1 C 1}\right) .
\end{gathered}
$$

The voltage vector diagram for the voltage reduction mode is shown in Fig. 3.

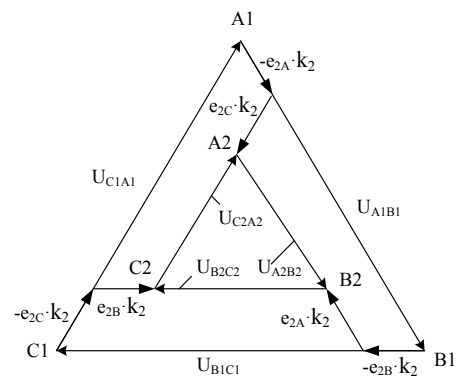

Fig. 3. Voltage vector diagram for the voltage reduction mode.

The mode of increasing the TVR output voltage is activated when the switches $\mathrm{TS}_{A}, \mathrm{TS} 8_{C}, \mathrm{TS} 5_{B}, \mathrm{TS} 6_{C}$, $\mathrm{TS} 7_{A}, \mathrm{TS}_{B}$ and switches TS3, TS4 of all phases are turned on. In this case, the EMF $\left(e_{2 A}, e_{2 B}, e_{2}\right)$ supplying the primary windings of the series transformer are reversed.

The shared use of longitudinal and transverse control modules allows to make a longitudinal-transverse regulation of the TVR output voltage.

The developed technical and circuit solutions formed the basis for the TVR prototype.

\section{Thyristor voltage regulator prototype}

The main technical characteristics of the TVR prototype are given in table 1 .

Table 1. Technical characteristics of the TVR

\begin{tabular}{|c|c|}
\hline Parameter & Value \\
\hline Nominal voltage (supply voltage) & $6 \mathrm{kV} \pm 10 \%$ \\
\hline $\begin{array}{c}\text { Variation range of the angle } \\
\text { voltage phase } \alpha\end{array}$ & $\pm 5^{\circ}$ \\
\hline $\begin{array}{c}\text { Measurement resolution of the } \\
\text { phase shift voltage } \alpha\end{array}$ & $1.5^{\circ}$ \\
\hline Range of regulation voltage & $\leq 10 \%$ \\
\hline $\begin{array}{c}\text { Regulation discreteness of the } \\
\text { voltage amplitude }\end{array}$ & $\leq 630 \mathrm{KVA}$ \\
\hline Load power & $106 \mathrm{kVA}$ \\
\hline Shunt-wound transformer power & $3 \times 28 \mathrm{kVA}$ \\
\hline Series transformer power
\end{tabular}

The TVR prototype is a container-type $6 \mathrm{kV}$ substation, consisting of $0.4 \mathrm{kV}$ longitudinal and transverse control thyristor modules, three-phase shunt 
and three single-phase series transformers and a $6 \mathrm{kV}$ switchgear. Fig. $4-6$ show the prototype appearance.

The TVR control system (CS) is two leveled. The first level CS (CS1) implements the physical execution of commands for the operational control of thyristors [21], carried out by the pulse-phase method. The CS1 is based on the algorithm of two-zone alternate regulation [22]. This method provides an output voltage change in the positive and negative power directions intervals, and also does not require the use of a current sensor. That allows to maintain the regulating properties of the TVR with a significant change in the value of the load current, as well as at idle.

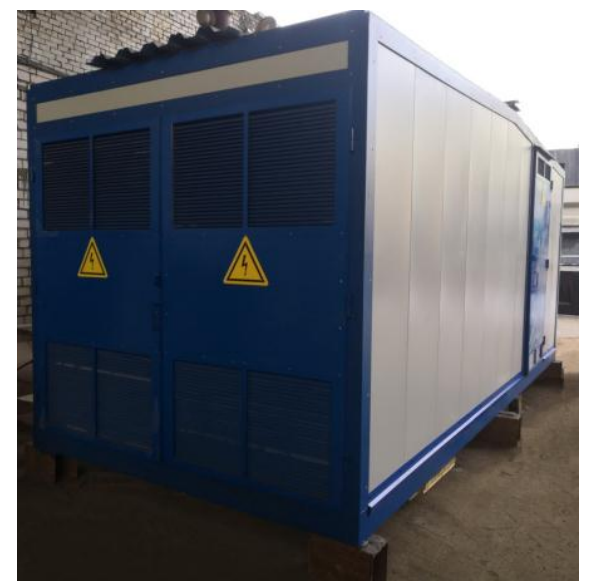

Fig. 4. TVR prototype

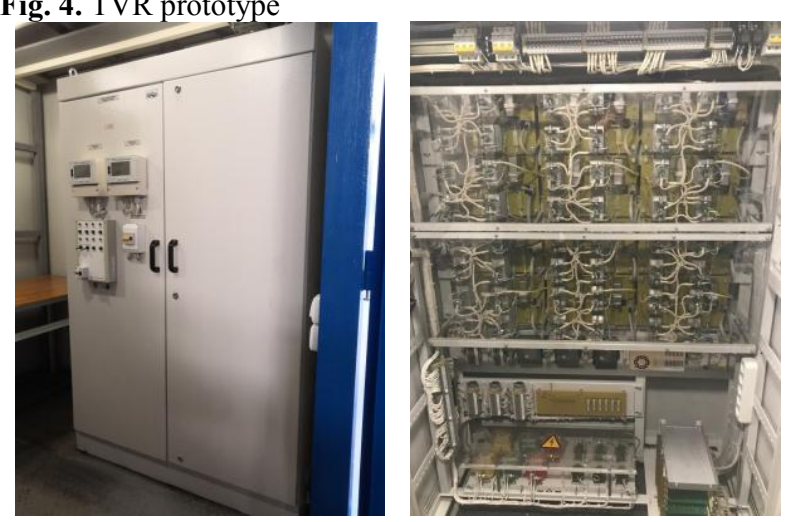

Fig. 5. Thyristor switch cabinet.

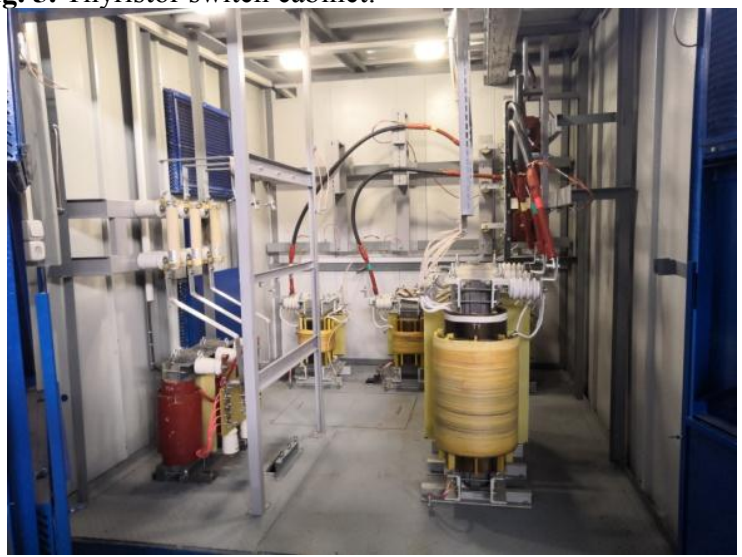

Fig. 6.Power transformer compartment.

The CS1 software part is implemented in the LabVIEW with Real Time and FPGA modules. Executable files provide the operation of the controller with a field programmable gate-array (FPGA), which generates and transmits control pulses to the TVR thyristors in the modes of transverse, longitudinal and longitudinal- transverse regulation.

Fig. 7 shows the main blocks of the CS1 software part.

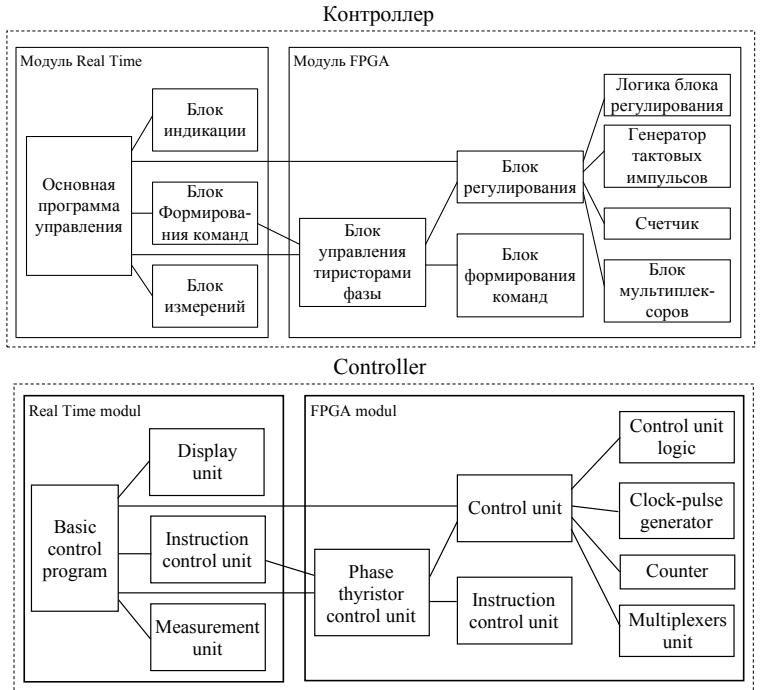

Fig. 7. Structure scheme of the CS1 software part

The CS1 hardware was implemented on three NI myRIO controllers. This was necessary to increase the speed of data processing. The controller carries out interaction between phases, as well as receiving and transmitting information to any external adaptive control system. The Real Time module provides measurements of frequency, power, $R M S$ voltages and currents. The $F P G A$ is the second hardware layer.

External commands as control actions are sent to the controller, which transmits them to the FPGA. Synchronizing pulses are formed at the FPGA level from the obtained voltage values of the high-voltage winding of the transformers. These pulses are generated at the moment of voltage polarity change when it crosses zero and are differentiated into four main synchronizing signals $U_{0}, U_{+}, U_{-}, U_{0+}$. Due to the commands received from the controller, pulses are formed in the FPGA to control the TVR thyristors. At the FPGA level, zero crossings are isolated from the incoming sinusoidal signal, analyzed when the sine is in a positive and negative state, and synchronizing pulses are formed when the sign changes from minus to plus $U_{0+}$.

Fig. 8 shows the control unit implementation.

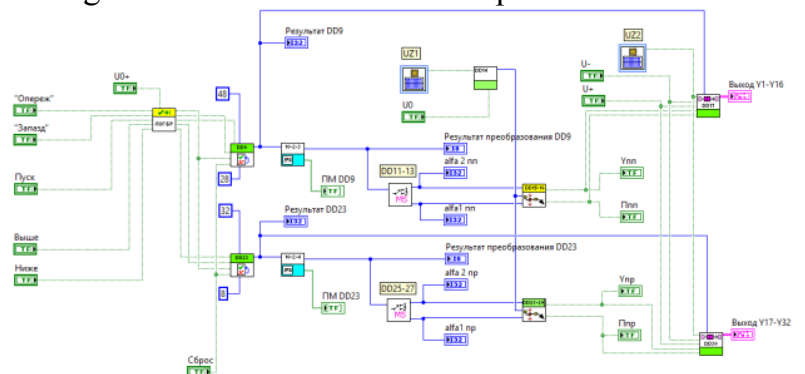

Fig. 8. Control unit of the CS1 
The TVR is controlled through a specialized interface of the control panel. The control panel is shown in Fig. 9.

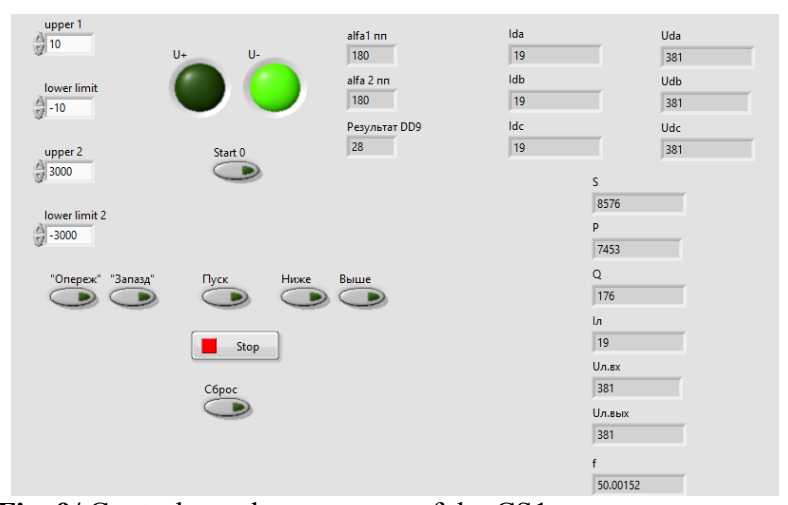

Fig. 9/ Control panel appearance of the CS1

The second level CS (CS2) performs the functions of centralized control and monitoring with the subsequent development of control commands for the CS1, as well as storage, transmission and remote access to information [23].

Fig. 10 shows CS2 functional diagram.

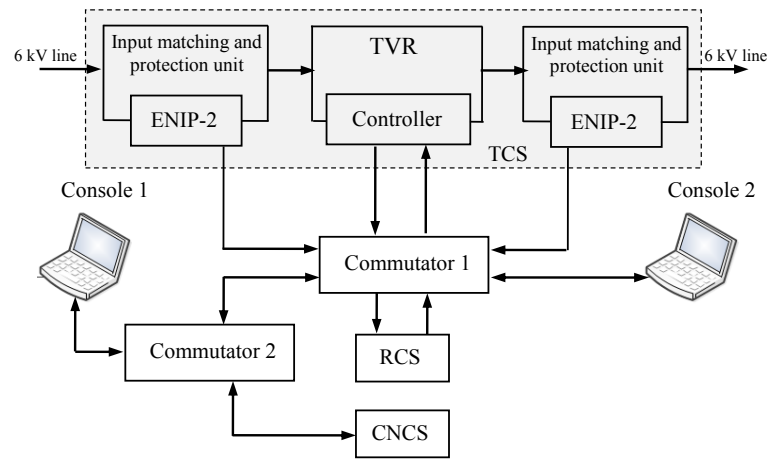

Fig. 10. Functional diagram of the CS2.

To the TVR prototype input and output measuring devices ENIP-2 are connected, it allows to calculate the RMS voltage, current and phase. The monitored parameters are transmitted to $\mathrm{CS} 2$ at a specified frequency using the Modbus TCP protocol.

The CS2 performs the functions of storage, processing of the received data and further transfer of analyzed parameters to the operator.

In addition, control actions are formed for the CS1. To do this, a connection is established between the CS1 and CS2 controllers via their own data transfer interface.

The CS2 contains two subsystems - CNCS (centralized network control system) and RCS (regulator control system). $X M L-R P C$ acts as a protocol for exchanging data and control signals in the RCS-CNCS network. The RCS console is used to control RCS configuration parameters.

The CNCS collects and processes information received from the RCS. The stored information can be visualized in the CNCS client and used to form control actions on the CS1.

The devices included in the CS2 are connected to a local area network with a switch via a high-speed
Ethernet connection. The CNCS system may be remote. For this, a communication channel was organized between the two commutators.

The operation of CS2 is based on the following algorithms:

- data aggregation algorithm received from RCS;

- network state determination and message delayed transmission algorithm;

- emergency situation tracking algorithm by threshold exceeding.

Experimental research was carried out to check the TVR prototype power equipment, control system and the device as a whole.

\section{Experimental research of thyristor voltage regulator prototype}

TVR prototype research was carried out according to the developed program and methods in four stages: power part testing, CS1, CS2 and prototype as a whole.

\subsection{Tests of the TVR prototype power equipment}

The following operations were performed when the TVR prototype power equipment testing:

- checking the connection diagrams (transformer and measuring equipment, thyristor switch unit);

- checking the integrity of conductors and semiconductor elements, as well as the reliability of contact connections (checking the insulation of conductors, the integrity of thyristor bodies, circuits of pulse amplifiers);

- checking the supply voltage polarity and magnitude of the boards of the pulse amplifiers;

- checking the local control console.

The following was found based on the results of power unit check:

- $6 \mathrm{kV}$ switchgear insulation resistance is at least $1000 \mathrm{M} \Omega, 0.4 \mathrm{kV}$ network insulation resistance is at least $1 \mathrm{M} \Omega$;

- $6 \mathrm{kV}$ and $0.4 \mathrm{kV}$ switchgear insulating strength with power frequency test voltage complies with the standards;

- no breakdowns and failures of the main circuits equipment were detected, the electrical circuits are functioning properly, the interlocks are in good order, and no damage has occurred that impedes their further work;

- contact resistance of detachable connections does not exceed $75 \mu \mathrm{Ohm}$;

- resistance of bolted or welded busbar joints does not exceed 1.2 times the resistance of a busbar section of the same length without joints.

\subsection{Tests of the CS1 TVR prototype}

The CS1 test consisted in checking the correctness of implementing the thyristor control algorithm and its functioning. 
The all control signals tracing was checked. The settings for the main and auxiliary modules of the program were calculated and stored in the read-only memory. Debugging of program blocks in case of emergency situations was performed.

The correctness of switching on thyristors in the transverse, longitudinal and longitudinal-transverse voltage regulation modes was checked.

The correctness of the control pulses formation at the moment of voltage zero crossing was checked.

Fig. 11 shows oscillograms of digital signals on thyristors during testing.

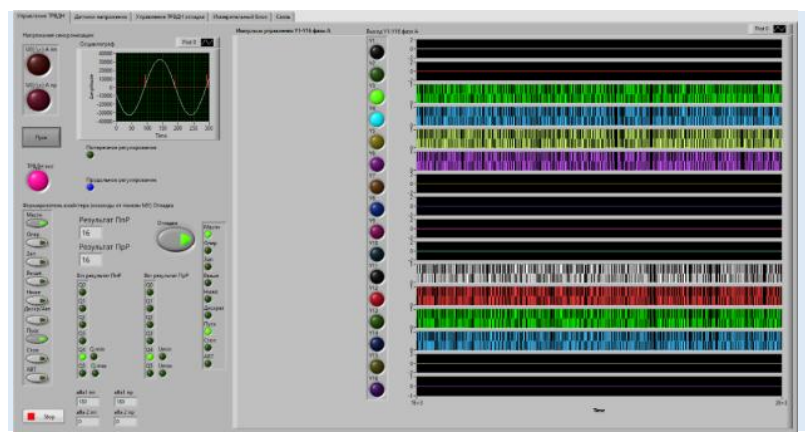

Fig. 11. Control panel of the CS1 controller during testing.

As a result of the research carried out, it was found that in the longitudinal and transverse regulation modes, control pulses by the control system are fed only to the thyristors which are embedded by the algorithm, it indicates the correct operation of the control system.

Fig. 12 shows an example of a control pulse shaping oscillogram.

Research has shown that a synchronizing pulse generated by a high voltage sensor appears at the moment the voltage sine wave crosses zero, which also corresponds to the control algorithm.

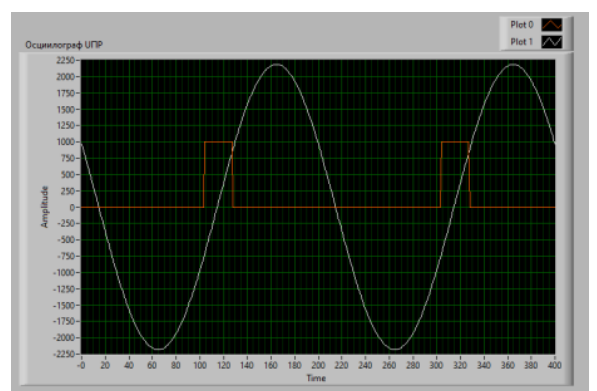

Fig. 12. Synchronizing pulses generated by the high voltage sensor when the voltage sine wave crosses zero from minus to plus.

\subsection{Tests of the CS2 TVR prototype}

The following was checked when CS2 testing:

- the main subsystems of the actions logic;

- interaction with an external monitoring and control system;

- user interaction;

- centralized collection, storage and processing of data; tracking equipment failures and communication channels;
- notifications of system users about failures;

- protection and authentication.

The signal generators were connected to the appropriate lines and generated signals with the parameters of voltage, current, phase shift within the range of acceptable values. On the service laptop, the console in which the values of the TVR parameters were monitored was launched, and control commands were sent to the CS1.

As a tests result the correctness of CS2 algorithms, the correctness of the executed and transmitted commands, as well as the transmitted and received data, were confirmed. The correctness of displaying the equipment status and communication lines was confirmed.

During the experiments, the correctness of the transmitted alarm messages, the ability to comment, acknowledge, filter, navigate from the message to the object that generated it were confirmed. The functions of displaying and exporting the history of archived alarms and creating sound and mail notifications about emergency events were also confirmed. The correctness of the receipt of sound and mail notifications about failures in communication channels and in equipment was confirmed.

\subsection{TVR prototype tests as a whole}

Experimental research of TVR prototype as a whole was carried out in the longitudinal, transverse and longitudinal-transverse voltage regulation modes.

Fig. 13 shows an example of the thyristor control pulses shaping oscillogram in the longitudinal regulation mode. When switching the TVR mode numbers, the duration of the control pulses changes, and the filling frequency of the pulses themselves is $10 \mathrm{kHz}$, which ensures the stability of the thyristor opening process.

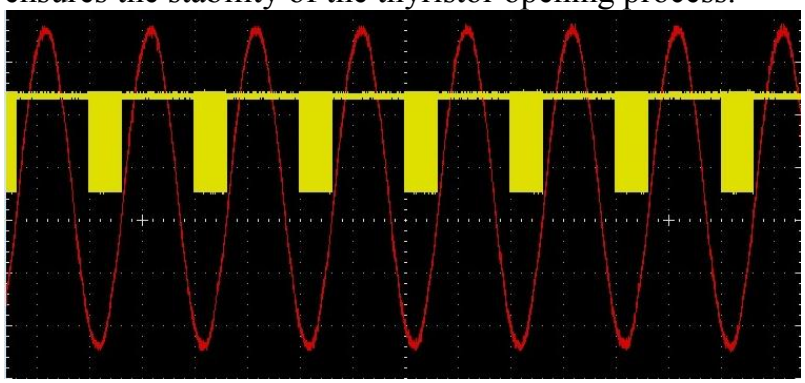

Fig. 13. Oscillogram of forming control pulses for thyristors and synchronizing voltage of $A$ phase in longitudinal regulation mode.

Fig. 14 shows the example of the TVR output voltages oscillogram in the longitudinal regulation mode. The analysis of the results showed that the regulation range of the output voltage relative to the input was \pm $10 \%$. The regulation discreteness of does not exceed $1.5 \%$. 


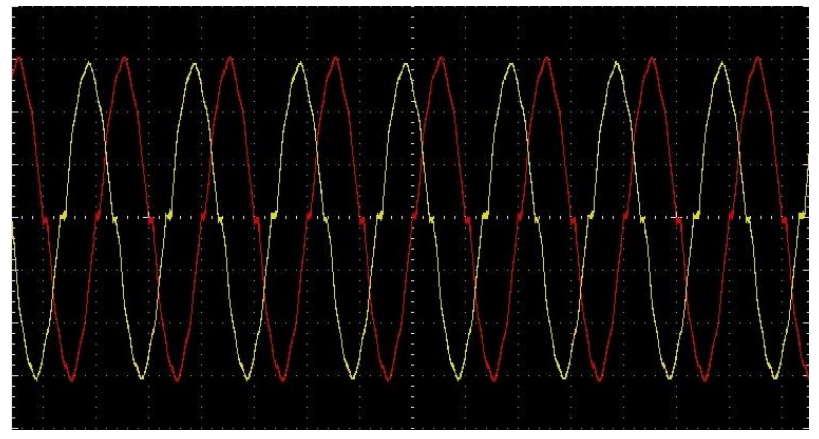

Fig. 14. Output voltages oscillogram of $A$ and $C$ phases in the longitudinal regulation mode (voltage reduction mode).

Fig. 15 shows the example of the TVR output voltages oscillogram in the transverse regulation mode. The Analysis of the results showed that the output voltage shift angle variation range relative to the input was $\pm 5^{\circ}$.

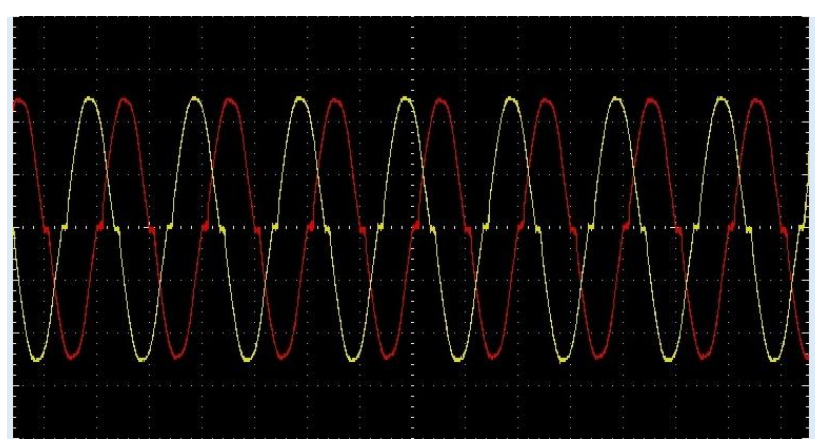

Fig. 15. Output voltages oscillogram of $A$ and $C$ phases in transverse regulation mode (delay mode).

Fig. 16 shows the example of the TVR output voltages oscillogram in the longitudinal-transverse regulation mode.

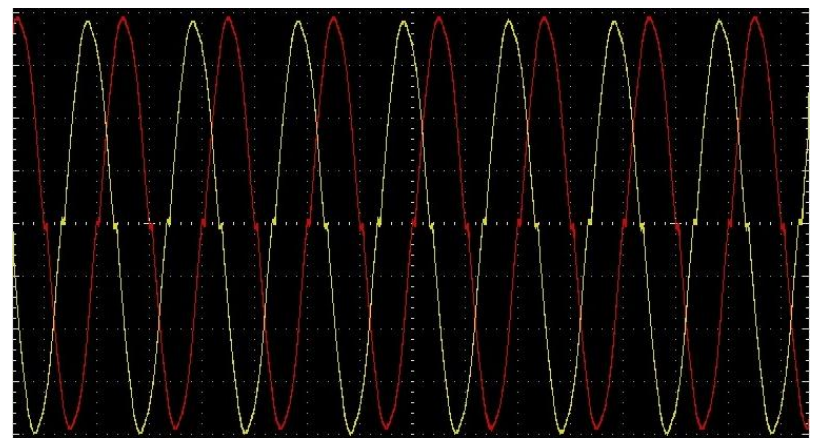

Fig. 16. Output voltages oscillogram of $A$ and $C$ phases in longitudinal-transverse regulation mode (reduction and delay mode).

The research results have shown the correctness of supplying thyristor control pulses in full accordance with the developed algorithm. It was found that the TVR ES provides smoothness and specified accuracy of voltage regulation in all modes.

\section{Conclusion}

The electric power systems building on the Internet of energy principle is accompanied by the need to implement flexible flow distribution in networks with a multi-circuit configuration and multiple power sources. The use of a thyristor voltage regulator allows this function to be carried out in $6-20 \mathrm{kV}$ power distribution networks.

The prototype of thyristor voltage regulator has been developed and manufactured, which makes it possible to exercise voltage control in the distribution electrical network, both in magnitude and in phase. It makes possible to redistribute power flows and optimize voltage at the load nodes.

Experimental research of power equipment, first and second level control systems (CS1 and CS2) and TVR prototype as a whole has been carried out.

The power equipment tests showed the correctness of the assembled connection schemes (thyristor switch unit, transformer and measuring equipment), as well as the reliability of contact connections.

A qualitative characteristic of the input and output signals was obtained when the CS1 testing. It was found that the thyristor control pulses were formed according to the developed algorithm.

The correctness of control system algorithms, executed and transmitted commands, transmitted and received data was confirmed as a result of the CS2 tests.

The TVR prototype research results indicated that the device provides smoothness and specified accuracy of voltage regulation in all modes.

The experimental research confirmed the TVR prototype operability and algorithms for its functioning, as well as the prototype readiness for trial operation

Research was supported by Russian Science Foundation grant (project No. 20-19-00541).

\section{References}

1. H. Hua, J. Cao Towards, 2019 IEEE International Conference on Energy Internet (ICEI), 573-578 (2019)

2. L. Cheng, N. Qi, F. Zhang, H. Kong, X. Huang, 2017 IEEE Conference on Energy Internet and Energy System Integration (EI2), 1-5 (2017)

3. V.A. Naumov, V.A. Matison, Energiya edinoy seti 2(44), 36-44 (2019)

4. N. Sakib, E. Hossain, S.I. Ahamed, IEEE Access 8, 69003-69037 (2020)

5. A.B. Loskutov, Smart electrical engineering 1(1), 927 (2018)

6. N.I. Voropay, M.V. Goubko, S.P. Kovalyov, L.V. Massel, D.A. Novikov, A.N. Raikov, S.M. Senderov, V.A. Stennikov, Probl. Upr. 1, 2-14 (2019)

7. F.V. Veselov, V.V. Dorofeev, Energy policy 5, 4352 (2018) 
8. K. Rakhi, Prerna, R. Chitrangada, Advances in Systems, Control and Automation 442, 647-655 (2017)

9. N.A. Hussein, A.A. Eisa, H.M. Mahmoud, S.A. Shehata, A. Othman, International Journal of Engineering \& Technology 7(3), 1656-1665 (2018)

10. P.P. Kumbhare, A.A. Nimje, P.R. Sawarkar, Silicon Photonics \& High Performance Computing. Advances in Intelligent Systems and Computing 718, 27-34 (2018)

11. A.V. Hardas, V. Rajderkar, D.V.K. Chandrakar, V.D. Hardas, Proc. 2018 International Conference on Smart Electric Drives and Power System (ICSEDPS), 132-136 (2018)

12. P. Kathal, A. Bhandakkar, International Journal for Research and Development in Engineering (IJRDE) 1(3), 82-91 (2013)

13. D. Ranamuka, A. P. Agalgaonkar, K. M. Muttaqi, IEEE Transactions on Sustainable Energy 5, 617628 (2014)

14. J. O. Petintin, M. Shaaban, 2014 IEEE Innovative Smart Grid Technologies - Asia (ISGT ASIA), 583588 (2014)

15. Y. Bot, A. Allali, International journal of renewable energy research 7(4), 1656-1662 (2017)

16. Y. Bot, A. Allali, International journal of renewable energy research 5(2), 581-585 (2015)

17. S. Wang, S. Chen, L. Ge, L. Wu, IEEE Transactions on Sustainable Energy 7(3), 1111-1123 (2016)

18. R. Yan, Y. Li, T.K. Saha, L.Wang, M.I. Hossain, IEEE Transactions on Smart Grid 9(3), 2224-2234 (2018)

19. P.V. Pullawar, S.R. Paraskar, S.S. Jadhao, International Journal of Innovative Research in Advanced Engineering 2(6), 164-168 (2015)

20. E. Sosnina, A. Asabin, A. Kralin, E. Kryukov, 2018 International Conf. on Smart Grid (icSmartGrid), 202-207 (2019)

21. A.A. Asabin, I.V. Belyanin, E.N. Sosnina, R.Sh. Bedretdinov, E.V. Kryukov, Smart electrical engineering 1(9), 25-39 (2020)

22. A.A. Asabin, RU Patent, 2398342 (2010)

23. E. Sosnina, R. Bedretdinov, E. Kryukov, A. Smirnov, 2019 IEEE Innovative Smart Grid Technologies - Asia (ISGT Asia), 1165-1169 (2019) 